\title{
A Market with a Social Consumption Externality
}

(Department of Economics Working Paper \#3)

James W. Friedman

Dept. of Economics, CB\# 3305

University of North Carolina

Chapel Hill, NC 27599-3305

USA

email: Jim_Friedman@unc.edu

Isabel Grilo

CORE, Université Catholique de Louvain

34, voie du Roman Pays

B-1348 Louvain-la-Neuve

Belgium

email: grilo@core.ucl.ac.be

January 12, 2001 


\begin{abstract}
We model a spatial market in which the utility of each consumer is affected by the collection of consumers who buy precisely the same product. The marginal contribution of another consumer $x$ 's purchase on consumer $y$ depends on $|y-x|$. This marginal contribution declines as $|y-x|$ increases and can be negative. This way of modeling preferences fits goods that signal something about a consumer's place in society; clothing styles, automobiles, and jewellry are examples. For a given number of firms and a given overall market size, we find the unique symmetric equilibrium and also derive comparative statics results on the optimal number of firms, the largest number of firms the market can support and the behavior of profits per firm as the number of firms increases.
\end{abstract}




\section{Introduction}

Every society has social stratification. Within a given society a member identifies more with some people than others and sees herself as being more like some people than others. Many consumption decisions are intended, consciously or subconsciously, to signal one's place in the social continuum. Clothing, hair styles, club memberships, automobiles, and housing neighborhoods all have some component of social identification associated with them. We present a model in this paper that is intended to capture consumption externalities of this sort. Placing a continuum of individuals and a finite number of goods in a spatial model, the utility of a consumer depends on the intrinsic value of the good purchased and on the particular collection of consumers who also buy the same good. Intrinsic utility depends on the price paid for the good and its distance from the consumer's location (i.e., the consumer's intrinsic ideal good). The addition to utility stemming from who else buys the same good depends on the similarity of the other consumers to buyer. The marginal contribution to utility by a specific other consumer is a decreasing function of the distance between them and, after some finite distance, can be negative.

The social identification can have either or both of two ingredients. One relates to jointness of consumption and the other to signalling. Imagine tennis clubs, golf clubs, and housing neighborhoods. Anyone choosing, for example, a tennis club to join is likely to prefer a club to which 'similar' people belong; that is, people who are similar in income level, education, and tastes. Within the club, the atmosphere is affected by the nature of the members and, in this sense, the services of the club are jointly consumed goods. As a rule, people feel more comfortable associating with others who are somewhat like them. Imagine a typical university professor who joins a club whose members are mainly upper level corporate executives. The professor is likely to feel like a fish out of water, as would the president of a Fortune 500 company in a tennis club whose members are mainly academics.

Casual observation reveals that men and women wear a large variety of hair styles. 
Imagine an investment banker who is considering whether to adopt spiked hair dyed magenta. Even if the banker found the hairstyle esthetically appealing, the kind of people who wear their hair that way are probably people with whom the banker would not wish to be identified. From a signalling standpoint, the style would be rejected in favor of something more commonly adopted in investment banking circles. Any good that either signals something about 'who' you are or that is, of necessity, jointly consumed with other buyers of the same thing, or both, is a good to which our model is addressed.

Network externalities and Veblenesque consumption externalities have been studied by economists have some resemblance to the externalities present in our model. Telephone systems provide a typical example of network externalities. As the number of telephone subscribers increases, the value of a subscription to a single person rises. Certain categories of computer software, such as word processors and spreadsheets, have the same feature, because many people wish to exchange files with others and such exchanges are facilitated by using the same software. At the present time there are three competing technologies in the United States for digital cellular telephone service. As a result the coverage of each system and the total subscriptions to all of them combined are undoubtedly smaller than if there were a single standard. In all of these examples the consumer cares, to some extent, who else buys the good; she is better off if friends and associates are buyers. Video formats provide a slightly different example, because numbers of buyers are primary. Exactly who else buys is not so important. If all buyers of videocassette recorders purchase the same system (e.g., VHS or beta), then the variety of films available for rent or purchase will be greater than if both systems share the market.

A good overview and many references to the network externalities literature can be found in [3]. Much of this literature is focused on technological issues, showing when a single standard will survive or when two standards can coexist. The number of technologies equals the number of firms. Generally, in the network externality 
models the more users of a good, the greater the value to any one user. In contrast to our model, which permits an aribitrary number of firms, the network externality literature typically deals with one or two. In network externality models consumers are anonymous; that is, the value of the good to one consumer depends on the total number of consumers without reference to their identity. In our model the identity of other consumers is critical; the contribution to Albert's utility when Betty consumes the same good can be positive or negative, depending on the similarity between the two of them.

Two papers dealing with Veblenesque consumption externalities are Bagwell and Bernheim [1] and Pesendorfer [4] who write about fashion goods. The former harks back to Veblen's [6] notion of conspicuous consumption, recognizing that some consumption choices play a vital signalling role, and is concerned with whether consumers will be willing to pay a higher price for a functionally equivalent variant of a good as a means of signalling higher wealth. In their model there are two types of household, one with high income and the other with low. Other things equal both wish to signal they are the high type. There are two functionally identical variants of a good that each might buy and the authors seek a separating equilibrium in which the high income consumers purchase the more expensive variant, sucessfully separating themselves from the low income consumers. The latter paper is concerned with cycles in fashion. Pesendorfer's point is that having a good that a few others, but not too many others, consume is most desirable. Again, the good plays a signalling role; however, the focus is on the monopolistic designer of the good who must decide on a price trajectory over time and on a frequency with which to introduce a new design. In terms of utility, the consumer's utility rises as the numbers consuming the same good increase from zero, but, at some modest level, continued increases diminish utility. With enough fellow consumers, the good is both exclusive and noticed. With too few it fails to be noticed and with too many it fails to be exclusive.

In both Bagwell and Bernheim [1] and Pesendorfer [4] the consumers are anony- 
mous; a consumer may care about how many others buy the same good, but not about precisely who they are. As with both of these papers, we interpret our consumers as being interested in signalling; however, we have a continuum of consumer types and an aribitrary number of goods and firms. In addition, our consumers place an intrinsic value on each good that varies across goods and across consumers. Specifically, the nearer a firm's location to a consumer, the higher is its intrinsic value to the consumer. Thus, we do not address the specific question that engages Bernheim and Bagwell or Pesendorfer. In the presence of many goods and of infinitely many consumer types, we focus on the equilibrium distribution of consumers among firms, on the number of firms that are viable at equilibrium, and on the welfare properties of equilibrium.

Consumers are not anonymous in our model. The utility of a consumer rises if any consumer within a specific distance buys the same good and fall if a consumer farther away buys it. The marginal contribution of consumer B to the utility of consumer $\mathrm{A}$ is a decreasing function of the distance between them. This corresponds to goods that signal the consumer's identity or goods that are consumed jointly with those who buy exactly the same thing. The consumer feels herself to be akin to those nearer and different from those who are far away. Professors dress differently than bankers and both dress differently than truck drivers. Typically, a person in one group would prefer to appear like someone else in the same group. Likewise, a person choosing a tennis or golf club is likely to prefer one that draws members from a similar background to her own.

The spatial model on the circle is described in Section 2. Then, in Section 3 the conditions for a local symmetric equilibrium are developed. The equilibrium is local, because the second order condition for profit maximization of a firm may not hold globally; however, we do have conditions under which the maximum is global. While a symmetric equilibrium is known to exist, it is not clear whether there are other equilibria. Intuition suggests not, but results are lacking. Section 4 examines some 
comparative statics properties of the model. The symmetric equilibrium profit per firm falls as the number of firms rises in a market of fixed overall size (i.e., on a circle of fixed circumference). In Section 5 the welfare optimal market size (number of firms) is found and a variant of the model is also briefly discussed in which the utility function has a somewhat different externality; a consumer's utility depends on the number of other consumers who buy the same good, but not on their identity or locations. The marginal utility due to an additional buyer is first positive and is decreasing, eventually becoming negative. This model has the same equilibrium conditions as the model we mainly analyze; however, the welfare optimal market size is smaller.

\section{The Model}

The market is modeled as a circle, as in Salop [5], with a finite number of firms

and a continuum of consumers. The details are spelled out in Section 2.1. The entire analysis is centered on characterizing symmetric equilibria in which all firms are equally spaced, choose the same price, and sell to markets of equal size. If the individual markets are too small (the number of firms is too large relative to the circumference of the circle), then each consumer may receive a rather large marginal benefit even from those consumers choosing the same good who are farthest away. In this case, consumers may be better off agglomerating into larger markets. Thus, the number of firms may not be robust. In Section 2.2 a condition on market size is found that ensures robustness of the number of firms.

\subsection{The Market and Consumer Utility}

There is a continuum of consumers uniformly distributed on a circle of circumference $(2 n+1) a$ with $2 n+1$ firms, $-n,-n+1, \ldots,-1,0,1, \ldots, n$. Firm 0 is located at 0 . The location of each other firm $k$ is the arc length from firm 0 to firm $k$ moving 
clockwise around the circle. Thus firm $k>0$ is at $k a$ and firm $k<0$ is at $(2 n+k+1) a$. The circle is not treated as having a fixed size; $a$, the distance between adjacent firms, is a parameter for which various values are considered. We consider only connected market areas for each firm; the market segment to which firm $i$ sells is the interval $\left[\underline{z}_{i}, \bar{z}_{i}\right)$. Each firm has zero variable costs and a fixed cost of $F$. Each consumer buys exactly one unit from only one firm, as long as doing so provides utility at least as large as the reservation utility of zero.

The location of a consumer $y$ on the circle indicates her ranking of goods as well as the degree of similarity between her and other consumers; her location corresponds to her ideal good. She has intrinsic utility $K$ for one unit of her ideal good. If she buys from firm $i$ at location $\ell_{i}$, her intrinsic utility is $K-t\left(y-\ell_{i}\right)^{2}$ where $t>0$. If $y \in\left[\underline{z}_{i}, \bar{z}_{i}\right)$ and firm $i$ 's price is $p_{i}$, then $y$ 's utility is her intrinsic utility minus the price paid and plus the externality effect. ${ }^{1}$ Thus,

$$
\begin{aligned}
u_{y}^{i}\left(p_{i}, \underline{z}_{i}, \bar{z}_{i}\right) & =K-p_{i}-t\left(y-\ell_{i}\right)^{2}+\int_{\underline{z}_{i}}^{\bar{z}_{i}}\left[\theta_{0}-\theta_{1}|y-z|\right] d z \\
& =K-p_{i}-t\left(y-\ell_{i}\right)^{2}+\theta_{0}\left(\bar{z}_{i}-\underline{z}_{i}\right)-\frac{\theta_{1}}{2}\left[\left(\bar{z}_{i}-y\right)^{2}+\left(y-\underline{z}_{i}\right)^{2}\right]
\end{aligned}
$$

The term $\int_{\underline{z}_{i}}^{\bar{z}_{i}}\left[\theta_{0}-\theta_{1}|y-z|\right] d z$ is the contribution to utility from the consumption externality. Another consumer $z$ who buys the same good as $y$ makes a marginal contribution to the utility of consumer $y$ in the amount of $\theta_{0}-\theta_{1}|y-z|$. The parameters $\theta_{0}$ and $\theta_{1}$ are both positive, so this marginal contribution is smaller, the larger is $|y-z|$ and, for sufficiently great $|y-z|$, it can be negative.

Denote by $p^{*}$ the symmetric equilibrium price. We assume $K$ is large enough that the whole market is always served at $p^{*}$. Then each firm sells to consumers up to a distance of $a / 2$ on both sides of its location. When the whole market is served at

\footnotetext{
${ }^{1}$ In Salop [5] utility is $u_{y}^{i}\left(p_{i}\right)=K-p_{i}-t\left|y-\ell_{i}\right|$; there are no externality effects and the $\left|y-\ell_{i}\right|$ term is linear rather than quadratic. In using the quadratic we follow d'Aspremont et al [2].
} 
equilibrium, $K$ satisfies

$$
K-p^{*}-\frac{t a^{2}}{4}+\theta_{0} a-\frac{\theta_{1} a^{2}}{2} \geq 0
$$

The left hand side of equation (2) is the utility of the least well off consumer buying from any given firm at a symmetric equilibrium. We assume the consumer's reservation utility is zero; therefore, equation (2) gives the condition on $K$ that ensures individual participation in the market by all consumers at a symmetric equilibrium.

\subsection{Minimum Spacing of Firms}

A candidate for a symmetric equilibrium, given by a price $p^{*}$ for all firms and equal markets of length $a$ with each firm at the center of its market, may not be robust to a positive measure of consumers switching firms if the interfirm distance $a$ is sufficiently small. In that case the externality effect may be so strong at the boundary between firms' markets that a positive measure of consumers at the boundary of one firm could increase each of their individual utilities by switching as a group to the neighboring firm. ${ }^{2}$ Their greater distance from the new firm would be more than compensated by the increase in the externality benefit. More precisely, suppose that the leftmost $\varepsilon$ of consumers of a firm switch to the firm on their left. If this brings an improvement to each individual in the deviating group, then the original consumer partition was not robust. The firm on the left now sells to an interval of length $a / 2$ on its left and $\varepsilon+a / 2$ on its right. The firm on the right sells to $a / 2-\varepsilon$ on its left and $a / 2$ on its right. Among the $\varepsilon$ measure of consumers who try the alternate firm, the one located at the new boundary has the lowest payoff from the new choice and the highest payoff from the old choice; therefore, if the change is profitable for her, it is profitable for

\footnotetext{
${ }^{2} \mathrm{~A}$ switch by only the one consumer at the boundary would have no effect on her utility because there would be no change in the externality term. A positive measure of consumers must switch to make an impact.
} 
all of them. Her payoff at the new firm is

$$
K-p-t\left(\frac{a}{2}+\varepsilon\right)^{2}+\int_{0}^{a+\varepsilon}\left[\theta_{0}-\theta_{1} z\right] d z
$$

and her payoff at the former firm, given that the other group members switch, would be

$$
K-p-t\left(\frac{a}{2}-\varepsilon\right)^{2}+\int_{0}^{a-\varepsilon}\left[\theta_{0}-\theta_{1} z\right] d z
$$

Not switching is better if equation (4) is greater than or equal to equation (3). The latter minus the former is

$$
2 \varepsilon\left(t a+\theta_{0}-\theta_{1} a\right)
$$

which is positive for any $\varepsilon>0$ if and only if

$$
a>\frac{\theta_{0}}{t+\theta_{1}}
$$

Equation (5) ensures that the symmetric market configuration is robust to any group deviation, because a group of measure $\varepsilon$ has a larger gain from deviation the nearer it is to the boundary. The analysis above shows that such a group, no matter what its size, cannot deviate profitably if equation (5) holds. Thus the minimum value of $a$ is $a=\theta_{0} /\left(t+\theta_{1}\right)$ and any larger value of $a$ is large enough to prevent a firm from being squeezed out.

\section{Equilibrium Conditions}

In this section we develop conditions for a symmetric local equilibrium, given the number and location of firms. At a symmetric equilibrium all firms charge the same price $p$ and no single firm could obtain a larger profit by using another price in a 
neighborhood of $p$. Due to symmetry the optimization conditions are identical for all firms, so, without loss of generality, attention here is devoted only to firm 0 which selects a price $p_{0}$. The value of $p_{0}$ affects all $2 n+1$ market boundaries. The conditions characterizing the boundaries between markets of all firms are determined in Section 3.1, given that all firms except firm 0 charge the same price $p$. Uniqueness of the relationship between $p_{0}$ and the market boundaries is established in Section 3.2, and the local symmetric equilibrium is characterized in Section 3.3.

\subsection{Market Boundaries}

As a preliminary to determining the exact relationship between $p, p_{0}$, and the market boundaries $\left(x_{0}, \ldots, x_{n}\right)$, the present section develops some basic equations involving these variables. With all firms other than firm 0 charging the same price, the market boundary between firms $i$ and $i+1$ for $i \geq 0$ will be exactly the same distance from the location of firm $i$ as the market boundary between firms $-i$ and $-(i+1)$. This permits the introduction of variables $x_{i}$ for $i=0, \ldots, n-1$ to represent the distance from firm $i$ to the market boundary between firms $i$ and $i+1$ as well as the distance from firm $-i$ to the market boundary between firms $-i$ and $-(i+1)$. See Figure 1 . Note that $x_{n}$, the distance of the consumer who is indifferent between firms $n$ and $-n$, to either firm is $a / 2$.

The location of the indifferent consumer between firm 0 and firm 1 is characterized by

$$
-p_{0}-t x_{0}^{2}+\int_{0}^{2 x_{0}}\left(\theta_{0}-\theta_{1} y\right) d y=-p-t\left(a-x_{0}\right)^{2}+\int_{0}^{a-x_{0}+x_{1}}\left(\theta_{0}-\theta_{1} y\right) d y
$$

The location of the indifferent consumer between firm $i$ and firm $i+1$ for $i=1, \ldots, n-$ 2 is characterized by

$$
-t x_{i}^{2}+\int_{0}^{a-x_{i-1}+x_{i}}\left(\theta_{0}-\theta_{1} y\right) d y=-t\left(a-x_{i}\right)^{2}+\int_{0}^{a-x_{i}+x_{i+1}}\left(\theta_{0}-\theta_{1} y\right) d y
$$




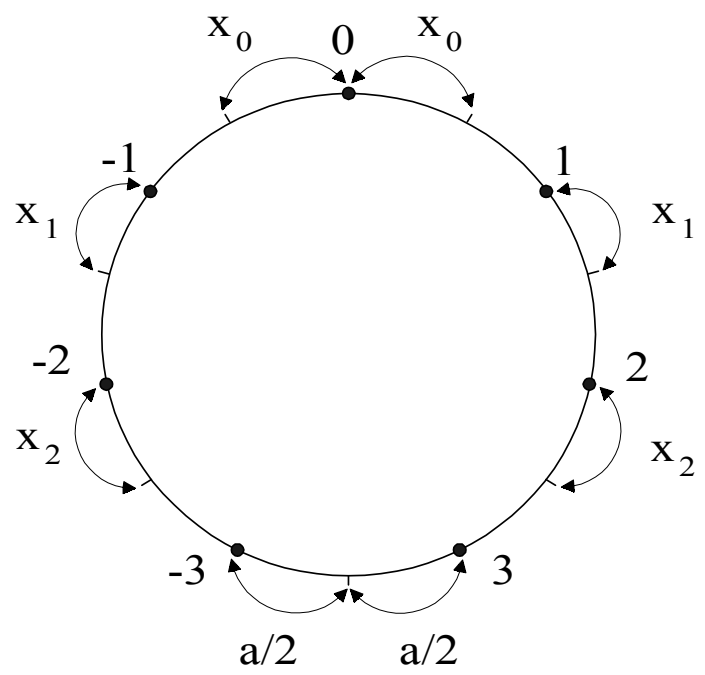

Figure 1: A Market with Boundaries

And the location of the indifferent consumer between firm $n-1$ and firm $n$ is characterized by

$$
-t x_{n-1}^{2}+\int_{0}^{a-x_{n-2}+x_{n-1}}\left(\theta_{0}-\theta_{1} y\right) d y=-t\left(a-x_{n-1}\right)^{2}+\int_{0}^{\frac{3}{2} a-x_{n-1}}\left(\theta_{0}-\theta_{1} y\right) d y
$$

The latter three equations can be rewritten, respectively, as

$$
\begin{gathered}
p_{0}=p+a t\left(a-2 x_{0}\right)-\left(a-3 x_{0}+x_{1}\right)\left[\theta_{0}-\frac{\theta_{1}}{2}\left(a+x_{0}+x_{1}\right)\right] \\
0=-a t\left(a-2 x_{i}\right)+\left(x_{i-1}-2 x_{i}+x_{i+1}\right)\left[\theta_{0}-\frac{\theta_{1}}{2}\left(2 a-x_{i-1}+x_{i+1}\right)\right] \\
0=-a t\left(a-2 x_{n-1}\right)+\left(x_{n-2}-2 x_{n-1}+\frac{a}{2}\right)\left[\theta_{0}-\frac{\theta_{1}}{2}\left(\frac{5 a}{2}-x_{n-2}\right)\right]
\end{gathered}
$$

Equation (8) can be subsumed into equation (7) because $x_{n}=a / 2$. Solving 
equation (7) for $x_{i}, i=1, \ldots, n-1$, yields

$$
x_{i}=\phi_{i}\left(x_{i-1}, x_{i+1}\right)=\frac{2 a^{2} t+\left(x_{i-1}+x_{i+1}\right)\left[\theta_{1}\left(2 a-x_{i-1}+x_{i+1}\right)-2 \theta_{0}\right]}{2\left[2 a t+\theta_{1}\left(2 a-x_{i-1}+x_{i+1}\right)-2 \theta_{0}\right]}
$$

\subsection{Market Allocations}

With the firms' locations given, the game they play consists of their simultaneously selecting prices. These prices jointly determine the market boundaries, $x=$ $\left(x_{0}, \ldots, x_{n}\right)$, and hence their profits. A change in $p_{0}$ will cause a change in all the market boundaries $x_{0}, \ldots, x_{n-1}$ except $x_{n}$. In this section we show that, given $p_{0}$ and $p$, the market allocation is uniquely determined. Lemma 2 establishes that for a given $x_{0} \in[0, a], p,\left(x_{1}, \ldots, x_{n}\right)$ is uniquely determined. Given $x_{0}$, the uniquely associated $\left(x_{1}, \ldots, x_{n}\right)$, and $p$, there is only one consistent price $p_{0}$ for firm 0 ; this price is given by equation (6).

Lemmas 1 and 2 show that the relationship between $x_{0}$ and $\left(x_{1}, \ldots, x_{n-1}\right)$ is unique as long as all firms other than firm 0 charge the same price $p$. The particular value of $p$ is immaterial. Lemma 1 shows that with $x_{n}=a / 2$ and for an arbitrary $x_{0} \in[0, a]$ equation $(9)$ for $i=1, \ldots, n-1$ defines a continuous function $\phi\left(\cdot ; x_{0}\right)=$ $\left(\phi_{1}, \ldots, \phi_{n-1}\right):[0, a]^{n-1} \rightarrow[0, a]^{n-1}$. Thus $\phi\left(\cdot ; x_{0}\right)$ must have a fixed point. Then Lemma 2 shows that $\phi\left(\cdot ; x_{0}\right)$ is a contraction, implying the fixed point is unique. Let $X \subset[0, a]^{n} \times\{a / 2\}$. A market allocation is $x=\left(x_{0}, \ldots, x_{n}\right) \in X$ that satisfies $\left(x_{1}, \ldots, x_{n-1}\right)=\phi\left(x_{1}, \ldots, x_{n} ; x_{0}\right)$, and $x_{n}=a / 2$. Denote by

$$
X^{M} \subset X=\left\{x: x_{0} \in[0, a], x_{n}=a / 2,\left(x_{1}, \ldots, x_{n-1}\right)=\phi\left(x_{1}, \ldots, x_{n-1} ; x_{0}\right)\right\}
$$

the set of market allocations.

Taking the two lemmas together, they show that for any $x_{0} \in[0, a]$ there is a unique associated market allocation $x \in X^{M}$. That is, given $x_{0}$, there is a unique $\left(x_{1}, \ldots, x_{n-1}\right)$ describing the location of the indifferent consumers between the pairs 
of firms $i$ and $i+1$ and $-i$ and $-i-1$ for $i=1, \ldots, n-1$. Equation (6) can then be regarded as a mapping from $X^{M} \times(0, \infty)$ to $\mathbb{R}_{+}$that maps pairs $(x, p)$ into a price $p_{0}$.

Lemma 1 If $a \geq 6 \theta_{0} /\left(2 t+3 \theta_{1}\right), x_{n}=a / 2$, and $x_{0} \in[0, a]$ there exists a market allocation $x=\left(x_{0}, \ldots, x_{n}\right)=\left[x_{0}, \phi\left(x_{1}, \ldots, x_{n-1} ; x_{0}\right), a / 2\right] \in[0, a]^{n+1}$.

Proof. It is readily seen that $a>\theta_{0} /\left(2 t+\theta_{1}\right)$ implies that the denominator of equation (9) is positive and bounded strictly away from zero, which, in view of the continuity of both numerator and denominator, implies that equation (9) is continuous. The bound $a>\theta_{0} /\left(2 t+\theta_{1}\right)$ is implied by $a \geq 6 \theta_{0} /\left(2 t+3 \theta_{1}\right)$.

To see that $x_{i-1}, x_{i+1} \in[0, a]$ implies $x_{i} \in[0, a]$, examine first the case of $\theta_{1}(2 a-$ $\left.x_{i-1}+x_{i+1}\right)-2 \theta_{0} \geq 0$. Here the condition holds trivially. When $\theta_{1}\left(2 a-x_{i-1}+x_{i+1}\right)-$ $2 \theta_{0}<0$,

$$
0 \leq \frac{2 a t+2 \theta_{1} a-4 \theta_{0}}{\left.2 a t+\theta_{1}\left(2 a-x_{i-1}+x_{i+1}\right)-2 \theta_{0}\right]}<x_{i}
$$

is sufficient for $x_{i} \geq 0$ and

$$
0 \leq 2 a^{2} t+\left(2 a-x_{i-1}-x_{i+1}\right)\left[\theta_{1}\left(2 a-x_{i-1}+x_{i+1}\right)-2 \theta_{0}\right]
$$

implies that $x_{i} \leq a$. To see that $x_{i} \geq 0$ note that the denominator in equation (10) is positive, so it is only necessary to ensure the numerator is not negative; that is, that $a \geq 2 \theta_{0} /\left(t+\theta_{1}\right)$. Then, using equation (11), $x_{i} \leq a$ is implied by $0 \leq 2 a t+2 \theta_{1} a-4 \theta_{0}$ or, equivalently, $a \geq 2 \theta_{0} /\left(t+\theta_{1}\right)$. It is readily verified that $2 \theta_{0} /\left(t+\theta_{1}\right)<6 \theta_{0} /\left(2 t+3 \theta_{1}\right) ;$ therefore, $\phi\left(\cdot ; x_{0}\right)$ is a continuous function from $[0, a]^{n-1}$ to $[0, a]^{n-1}$. From the Brouwer fixed point theorem $\phi\left(\cdot ; x_{0}\right)$ has a fixed point which corresponds to a market allocation $\left(x_{0}, \phi\left(\cdot ; x_{0}\right), a / 2\right) \in X^{M}$. 
The derivatives of equation (9) for $i=1, \ldots, n-1$ are

$$
\begin{gathered}
\frac{\partial \phi_{i}}{\partial x_{i-1}}=\frac{\partial x_{i}}{\partial x_{i-1}}=\frac{\left[\theta_{1}\left(2 a-x_{i-1}+x_{i+1}\right)-2 \theta_{0}\right]^{2}+2 a t\left[\theta_{1}\left(3 a-2 x_{i-1}\right)-2 \theta_{0}\right]}{2\left[2 a t+\theta_{1}\left(2 a-x_{i-1}+x_{i+1}\right)-2 \theta_{0}\right]^{2}} \\
\frac{\partial \phi_{i}}{\partial x_{i+1}}=\frac{\partial x_{i}}{\partial x_{i+1}}=\frac{\left[\theta_{1}\left(2 a-x_{i-1}+x_{i+1}\right)-2 \theta_{0}\right]^{2}+2 a t\left[\theta_{1}\left(3 a-2 x_{i-1}\right)-2 \theta_{0}\right]}{2\left[2 a t+\theta_{1}\left(2 a-x_{i-1}+x_{i+1}\right)-2 \theta_{0}\right]^{2}}
\end{gathered}
$$

except that $\partial \phi_{i} / \partial x_{i+1}=0$ for $i=n-1$, because $x_{n}=a / 2$.

Lemma 2 If $a \geq 6 \theta_{0} /\left(2 t+3 \theta_{1}\right), x_{n}=a / 2$, and $x_{0} \in[0, a]$ the function $\phi\left(\cdot ; x_{0}\right)$ is a contraction and hence the market allocation $x=\left(x_{0}, \phi\left(\cdot ; x_{0}\right), a / 2\right) \in X^{M}$ associated with $x_{0} \in[0, a]$ is unique.

Proof. When both $\partial x_{i} / \partial x_{i-1}$ and $\partial x_{i} / \partial x_{i+1}$ are in the interval $(-0.5,0.5)$, the function $\phi\left(\cdot ; x_{0}\right)$ is a contraction; a contraction mapping from a set into itself has a unique fixed point.

To verify the contraction property, $\partial x_{i} / \partial x_{i-1}<0.5$ and $\partial x_{i} / \partial x_{i-1}>-0.5$ are checked separately. Proof for $\partial x_{i} / \partial x_{i+1}$ is parallel and will be omitted. It is easily seen that $\partial x_{i} / \partial x_{i-1}<0.5$ is equivalent to

$$
0<2 a t+\theta_{1}\left(a+2 x_{i+1}\right)-2 \theta_{0}
$$

which is ensured by $a>2 \theta_{0} /\left(2 t+\theta_{1}\right)$. That $-0.5<\partial x_{i} / \partial x_{i-1}$ is equivalent to

$$
\begin{array}{ll} 
& -4 a^{2} t^{2}-4 a t\left[\theta_{1}\left(2 a-x_{i-1}+x_{i+1}\right)-2 \theta_{0}\right]-\left[\theta_{1}\left(2 a-x_{i-1}+x_{i+1}\right)-2 \theta_{0}\right]^{2} \\
< & {\left[\theta_{1}\left(2 a-x_{i-1}+x_{i+1}\right)-2 \theta_{0}\right]^{2}+2 a t\left[\theta_{1}\left(3 a-2 x_{i-1}\right)-2 \theta_{0}\right]}
\end{array}
$$

The latter is implied by

$$
-4 a^{2} t^{2}-4 a t\left[\theta_{1}\left(2 a-x_{i-1}+x_{i+1}\right)-2 \theta_{0}\right] \leq 2 a t\left[\theta_{1}\left(3 a-2 x_{i-1}\right)-2 \theta_{0}\right]
$$


which, in turn, is implied by $a \geq 6 \theta_{0} /(2 t+3 \theta)$. Finally, $6 \theta_{0} /(2 t+3 \theta)>2 \theta_{0} /\left(2 t+\theta_{1}\right)$ which establishes the contraction property and completes the proof.

In view of Lemma 2 there is a unique function $\omega:[0, a] \rightarrow[0, a]^{n-1}$ that satisfies $\left(x_{0}, \omega\left(x_{0}\right), a / 2\right)=\left(x_{0}, \omega_{1}\left(x_{0}\right), \ldots, \omega_{n-1}\left(x_{0}\right), a / 2\right) \in X^{M}$ for all $x_{0} \in[0, a]$. That is, for each $x_{0} \in[0, a], \omega\left(x_{0}\right)=\left(\omega_{1}\left(x_{0}\right), \ldots, \omega_{n-1}\left(x_{0}\right)\right)$ is the unique fixed point of $\phi\left(\cdot ; x_{0}\right)$. We will henceforth assume

$$
a>\frac{6 \theta_{0}}{2 t+3 \theta_{1}}
$$

The bound in equation (14), from Lemma 2, is larger than the bound in equation (5) which ensures that no firm can be squeezed out of the market due to being too closely spaced.

\subsection{Symmetric Equilibrium}

In this section the first and second order conditions for profit maximization for firm 0 are developed. As this is done under symmetric conditions, it will determine the profit maximization conditions characterizing a symmetric equilibrium for the market. For technical reasons it is easier to treat $x_{0}$ as the decision variable rather than $p_{0}$.

From equation (6) and Lemma 2 the profit of firm 0 can be expressed entirely as a function of $x_{0}$ and $p$ using the function $\omega$. Then the value of $x_{0}$ that maximizes profit can be found. Evaluating the first order condition for profit maximization requires that the first derivative of $\omega_{1}$, evaluated at $x_{0}=a / 2$, be found. This is done in Lemma 3. Then the equilibrium price and the second order condition are evaluated in Theorem 1. Obtaining the equilibrium price after having Lemma 3 is easy; however, determining the second order condition, which requires knowing the second derivative of $\omega_{1}$ evaluated at $x_{0}=a / 2$, is more difficult. 
Using $\omega_{1}$ equation (6) can be expressed as the function $P\left(p, x_{0}\right)$ :

$$
\begin{aligned}
P\left(p, x_{0}\right)= & p+\left(\theta_{1} a-\theta_{0}\right) \omega_{1}\left(x_{0}\right)-\left(2 a t+\theta_{1} a-3 \theta_{0}\right) x_{0} \\
& +\frac{a\left(2 a t+\theta_{1} a-2 \theta_{0}\right)-\theta_{1}\left[3 x_{0}-\omega_{1}\left(x_{0}\right)\right]\left[x_{0}+\omega_{1}\left(x_{0}\right)\right]}{2}
\end{aligned}
$$

Profit maximization for firm 0 can be conceived as choosing $x_{0} \in[0, a]$, because, once $x_{0}$ is selected, $x_{1}=\omega_{1}\left(x_{0}\right)$, and the values of $x_{0}, x_{1}$, and $p$ together determine uniquely the value of $p_{0}$ (subject to the boundary condition that $p_{0} \geq 0$ ). The first order condition for an interior profit maximum is

$$
\begin{aligned}
\frac{d \pi_{0}}{d x_{0}} & =2 P+2 x_{0}\left(\frac{\partial P}{\partial x_{0}}+\frac{\partial P}{\partial x_{1}} \frac{d x_{1}}{d x_{0}}\right) \\
& =2 P+2 x_{0}\left(\frac{\partial P}{\partial x_{0}}+\frac{\partial P}{\partial x_{1}} \omega_{1}^{\prime}\left(x_{0}\right)\right)=0
\end{aligned}
$$

The partial derivatives in equation (16) are straightforward to calculate; however $\omega_{1}^{\prime}$ is rather more difficult and is taken up in Lemma 3.

Lemma 3 Assume $a \geq 6 \theta_{0} /\left(2 t+3 \theta_{1}\right)$. Then, at a symmetric equilibrium, (i)

$$
\frac{\partial \phi_{i}}{\partial x_{i+1}}=\frac{\partial x_{i}}{\partial x_{i+1}}=\frac{\theta_{1} a-\theta_{0}}{2\left(a t+\theta_{1} a-\theta_{0}\right)} \equiv \mu
$$

and (ii)

$$
\omega_{i}^{\prime} \equiv \frac{d x_{i}}{d x_{0}}=\frac{r_{2}^{n-i}-r_{1}^{n-i}}{r_{2}^{n}-r_{1}^{n}} \in(-1,1)
$$

where

$$
r_{1}=\frac{1}{2 \mu}-\sqrt{\frac{1}{4 \mu^{2}}-1} \quad \text { and } \quad r_{2}=\frac{1}{2 \mu}+\sqrt{\frac{1}{4 \mu^{2}}-1}
$$


In addition, $\omega_{i}^{\prime}$ increases in absolute value as $n$ increases, falls in absolute value as $i$ increases, and, for $\mu>0, \omega_{i}^{\prime}$ is always positive and

$$
\lim _{n \rightarrow \infty} \omega_{i}^{\prime}=r_{1}^{i}
$$

while, for $\mu<0, \omega_{i}^{\prime}$ alternates in sign and

$$
\lim _{n \rightarrow \infty} \omega_{i}^{\prime}=r_{2}^{i}
$$

Proof. Item (i) is proved by setting $x_{i-1}=x_{i+1}=a / 2$ in equation (9) and simplifying.

In proving item (ii) note that Lemma 2 establishes that $\mu \in(-0.5,0.5)$. We evaluate $\omega_{i}^{\prime}$, using equation (9). Observe that

$$
d x_{i}=\frac{\partial x_{i}}{\partial x_{i-1}} d x_{i-1}+\frac{\partial x_{i}}{\partial x_{i+1}} d x_{i+1}
$$

and that $d x_{n}=0$. Thus we may write

$$
\left[\begin{array}{ccccc}
-1 & \frac{\partial x_{1}}{\partial x_{2}} & 0 & \cdots & 0 \\
\frac{\partial x_{2}}{\partial x_{1}} & -1 & \frac{\partial x_{2}}{\partial x_{3}} & \cdots & 0 \\
\vdots & \vdots & \ddots & \vdots & \vdots \\
0 & \cdots & \frac{\partial x_{n-2}}{\partial x_{n-3}} & -1 & \frac{\partial x_{n-2}}{\partial x_{n-1}} \\
0 & \cdots & 0 & \frac{\partial x_{n-1}}{\partial x_{n-2}} & -1
\end{array}\right]\left[\begin{array}{c}
d x_{1} \\
d x_{2} \\
\vdots \\
d x_{n-2} \\
d x_{n-1}
\end{array}\right]=\left[\begin{array}{c}
d x_{0} \\
0 \\
\vdots \\
0 \\
0
\end{array}\right]
$$

Evaluating equation (21) at a symmetric equilibrium, all the partial derivatives are given by equation (18). From equation (20) evaluated at equilibrium we see

$$
\omega_{i}^{\prime}=\mu \omega_{i-1}^{\prime}+\mu \omega_{i+1}^{\prime}
$$


for $i=1, \ldots, n-1$. Since $d x_{0} / d x_{0}=1$ this means

$$
\omega_{1}^{\prime}=\mu+\mu \omega_{2}^{\prime}
$$

and by construction $d x_{n} / d x_{0}=0$, because $x_{n}=a / 2$ is a constant so that

$$
\omega_{n-1}^{\prime}=\mu \omega_{n-2}^{\prime}
$$

To see that equation (18), holds, suppose that $\omega_{i}^{\prime}=r^{i}$ for some, as yet undetermined, parameter $r$. Equation (22) implies $r=\mu\left(1+r^{2}\right)$. Solving the latter quadratic for $r$ yields the roots $r_{1}$ and $r_{2}$ from equation (19). It is easily verified that $\mu \in[-0.5,0.5]$ implies the roots are positive and real. If $\mu= \pm 0.5$ there is only one root. Furthermore, $r_{1} r_{2}=1$ and $r_{1}+r_{2}=1 / \mu$. All solutions to the system of equations (22) have the form

$$
\omega_{i}^{\prime}=A r_{1}^{i}+B r_{2}^{i}
$$

where the constants $A$ and $B$ are determined by equations (23) and (24) from which we see

$$
A=\frac{r_{2}^{n}}{r_{2}^{n}-r_{1}^{n}} \quad \text { and } \quad B=\frac{-r_{1}^{n}}{r_{2}^{n}-r_{1}^{n}}
$$

Using equation (25) in equation (26) yields equation (18). It is clear from inspection of equation $(18)$ that $\omega_{i}^{\prime} \in(-1,1)$.

For $\mu>0, r_{2}>1>r_{1}>0$ and for $\mu<0, r_{2}<-1<r_{1}<0$; therefore, the sign of $\omega_{i}^{\prime}$ is always positive for $\mu>0$ and alternates, being positive for even $i$, for $\mu<0$. As $n$ increases $\omega_{i}^{\prime}$ increases in absolute value, converging to $r_{1}^{i}$ for $\mu>0$ and to $-r_{2}^{i}$ for $\mu<0$.

Note that $a t+\theta_{1} a-\theta_{0}>0$ if $a \geq 6 \theta_{0} /\left(2 t+3 \theta_{1}\right)$. Whether $\mu$ is positive or 
negative thus depends on the sign of $\theta_{1} a-\theta_{0}$. Lemma 3 tells us that when $\mu>0$ the derivatives $\omega_{i}^{\prime}$ are strictly positive, monotone decreasing in $i$, and $\omega_{1}^{\prime}<1$. This means that, as $x_{0}$ increases from $a / 2$, the market served by firm 0 increases, that of all other firms decrease, and the decrease is smaller for firm $i$ than for firm $i-1$. Curiously, when $\mu<0$ the derivatives $\omega_{i}^{\prime}$ alternate in sign and decrease in absolute value starting with $-1<\omega_{1}^{\prime}<0$. If $x_{0}$ increases, the market allocations of even numbered firms get larger, those of odd numbered firms get smaller, and the $x_{i}$ alternate in being below and above $a / 2$; however, $\left|x_{i}-a / 2\right|$ is montone decreasing in $i$ for $i=1, \ldots, n-1$.

Theorem 1 below characterizes the market price at a local symmetric equilibrium. The equilibrium is local in the sense that the first and second derivatives are evaluated only at the equilibrium; therefore, we cannot be certain whether firm 0 would obtain higher profit at some price lying outside a neighborhood of the local equilibrium.

Theorem 1 The price at a local symmetric equilibrium is

$$
p=\frac{a}{2}\left[2 a t+3 \theta_{1} a-3 \theta_{0}-\left(\frac{\theta_{1} a}{3}-\theta_{0}\right) \frac{r_{2}^{n-1}-r_{1}^{n-1}}{r_{2}^{n}-r_{1}^{n}}\right]>0
$$

Proof. Without loss of generality attention can be restricted to firm 0. Consider the first order condition at the symmetric equilibrium,

$$
\begin{aligned}
\frac{d \pi_{0}}{d x_{0}} & =2 P+2 x_{0}\left(\frac{\partial P}{\partial x_{0}}+\frac{\partial P}{\partial x_{1}} \frac{d x_{1}}{d x_{0}}\right) \\
& =2 p+a\left[3 \theta_{0}-2 a t-3 \theta_{1} a+\left(\frac{\theta_{1} a}{3}-\theta_{0}\right) \frac{r_{2}^{n-1}-r_{1}^{n-1}}{r_{2}^{n}-r_{1}^{n}}\right]=0
\end{aligned}
$$

and solve for the equilibrium price, which is given by equation (27). Inspection of equation (27) reveals that it must be positive if $a>6 \theta_{0} /\left(2 t+3 \theta_{1}\right){ }^{3}$

The second order condition, which we evaluate at the equilibrium price obtained

\footnotetext{
${ }^{3}$ Three cases must be separately examined for $\theta_{0} \geq \theta_{1} a, \theta_{1} a>\theta_{0}>\theta_{1} a / 3$, and $\theta_{1} a / 3 \geq \theta_{0}$.
} 
from equation (28), is

$$
\begin{aligned}
\frac{d^{2} \pi_{0}}{d x_{0}^{2}}= & 4\left(\frac{\partial P}{\partial x_{0}}+\frac{\partial P}{\partial x_{1}} \omega_{1}^{\prime}\right) \\
& +2 x_{0}\left(\frac{\partial^{2} P}{\partial x_{0}^{2}}+2 \frac{\partial^{2} P}{\partial x_{0} \partial x_{1}} \omega_{1}^{\prime}+\frac{\partial^{2} P}{\partial x_{1}^{2}}\left(\omega_{1}^{\prime}\right)^{2}\right) \\
& +2 x_{0} \frac{\partial P}{\partial x_{1}} \omega_{1}^{\prime \prime}
\end{aligned}
$$

The first two of the three terms are clearly negative and we have exact expressions for them. The third term is problematic. Using equation (28) and $a / 2=x_{0}$ at equilibrium, the first term is $-8 p / a$ and the second term is

$$
-a\left(3 \theta_{1}+2 \theta_{1} \omega_{1}^{\prime}+\frac{\theta_{1}}{3}\left(\omega_{1}^{\prime}\right)^{2}\right)
$$

The sign of the first term is clear from $p$ being positive and the sign of the second term is clear from $\left|\omega_{1}^{\prime}\right|<1$.

We turn now to obtaining an expression for $\omega_{1}^{\prime \prime}$. We know that

$$
\omega_{i}^{\prime}=\phi_{i-1}\left(x_{i-1}, x_{i+1}\right) \omega_{i-1}^{\prime}+\phi_{i+1}\left(x_{i-1}, x_{i+1}\right) \omega_{i+1}^{\prime} \text { for } i=1, \ldots, n-1
$$

with the understanding that $\omega_{0}^{\prime} \equiv 1$ and $\omega_{n}^{\prime} \equiv 0$. From the previous expression we can write

$$
\begin{aligned}
\omega_{i}^{\prime \prime}= & \phi_{i-1} \omega_{i-1}^{\prime \prime}+\omega_{i-1}^{\prime}\left(\frac{\partial \phi_{i-1}}{\partial x_{i-1}} \omega_{i-1}^{\prime}+\frac{\partial \phi_{i-1}}{\partial x_{i+1}} \omega_{i+1}^{\prime}\right) \\
& +\phi_{i+1} \omega_{i+1}^{\prime \prime}+\omega_{i+1}^{\prime}\left(\frac{\partial \phi_{i+1}}{\partial x_{i-1}} \omega_{i-1}^{\prime}+\frac{\partial \phi_{i+1}}{\partial x_{i+1}} \omega_{i+1}^{\prime}\right)
\end{aligned}
$$

At equilibrium

$$
\frac{\partial \phi_{i-1}}{\partial x_{i+1}}=\frac{\partial \phi_{i+1}}{\partial x_{i-1}}=0
$$


and

$$
\frac{\partial \phi_{i+1}}{\partial x_{i+1}}=-\frac{\partial \phi_{i-1}}{\partial x_{i-1}}=\frac{a^{2} t \theta_{1}}{4\left(a t+\theta_{1} a-\theta_{0}\right)^{2}} \equiv c
$$

Recall that at equilibrium $\phi_{i-1}=\phi_{i+1}=\mu$ (see equation (17)). Thus at equilibrium equation (30) can be rewritten

$$
\omega_{i}^{\prime \prime}=\mu\left(\omega_{i-1}^{\prime \prime}+\omega_{i+1}^{\prime \prime}\right)+L_{i}
$$

where

$$
L_{i}=c\left[\left(\omega_{i+1}^{\prime}\right)^{2}-\left(\omega_{i-1}^{\prime}\right)^{2}\right] \text { for } i=1, \ldots, n-1
$$

Clearly $L_{i}<0$ because $\left|\omega_{i+1}^{\prime}\right|<\left|\omega_{i-1}^{\prime}\right|<1$. Denote by $J_{j}(\mu)=\mu^{j}\left(r_{2}^{j+1}-r_{1}^{j+1}\right) /\left(r_{2}-\right.$ $\left.r_{1}\right)$ the determinant of

$$
\left[\begin{array}{ccccc}
1 & -\mu & 0 & \cdots & 0 \\
-\mu & 1 & -\mu & \cdots & 0 \\
\vdots & \vdots & \ddots & \vdots & \vdots \\
0 & \cdots & -\mu & 1 & -\mu \\
0 & \cdots & 0 & -\mu & 1
\end{array}\right]
$$

where the matrix is of order $j$ and $r_{1}$ and $r_{2}$ are defined in equation (19). Then

$$
\omega_{1}^{\prime \prime}=\frac{\sum_{i=1}^{n-1} \mu^{i-1} L_{i} J_{n-i-1}(\mu)}{J_{n-1}(\mu)}=\frac{1}{\mu} \sum_{i=1}^{n-1} L_{i} \frac{r_{2}^{n-i}-r_{1}^{n-i}}{r_{2}^{n}-r_{1}^{n}}=\frac{1}{\mu} \sum_{i=1}^{n-1} L_{i} \omega_{i}^{\prime}
$$

The sign of equation (31) when $\mu>0$ is negative, because $\omega_{i}^{\prime}>0$ and $L_{i}<0$ for all $i$. The sign of the third term in equation (29) is difficult to determine; however, this term goes to zero as $t$ goes to zero. This can be seen by noting that $c$ goes to zero as $t$ goes to zero and everything else is finite. Meanwhile the second term is bounded 
strictly away from zero. Consequently, there must be a non-empty interval $\left(0, t^{*}\right)$ of values of $t$ within which the second order condition holds.

The lower bound on $K$ can be computed by using the equilibrium price from equation (27) in equation (2).

In Theorem 1 the second derivative is evaluated at the equilibrium price only and there is not sufficient information to know if it remains negative throughout the domain of $x_{0}$. Consequently, it is not entirely clear that $x_{0}=a / 2$ is a global maximum over $x_{0} \in[0, a]$ rather than merely a maximum over some, possibly small, open neighborhood of $a / 2$. This issue can be partially addressed by analyzing a special case of the model with $\theta_{1}=0$. Then the functions $\omega_{i}$ are affine and their slope coefficients are given by equation (25). If at $>2 \theta_{0}$ then $\mu \in(-0.5,0)$ and the second order condition reduces to

$$
\frac{d^{2} \pi_{0}}{d x_{0}^{2}}=4\left(\frac{\partial P}{\partial x_{0}}+\frac{\partial P}{\partial x_{1}} \omega_{1}^{\prime}\right)<0
$$

which is constant and bounded strictly away from zero. Thus for $\theta_{1}=0$ the first order condition (28) is a global maximum and, due to continuity of the derivatives of $\pi_{0}$, equation (28) corresponds to a global maximum for some non-empty interval $\theta_{1} \in\left[0, \bar{\theta}_{1}\right)$. See Appendix A for details.

\section{Comparative Statics}

In Lemma 4 we see that the equilibrium price is monotone as a function of $n$; however, this function can be either monotone increasing or decreasing, depending on the sign of $\theta_{0}-\theta_{1} a / 3$. Supposing the firms have a fixed entry cost of $F$, we can determine the number of firms at a free entry equilibrium with minimal non-negative profit. Lemma 4 shows that the condition $p a-F=0$ implicitly defines a function $n=$ $N\left(a, t, \theta_{0}, \theta_{1}, F\right)$ whose characteristics are examined in Lemma 6 . Denoting by $p$ the 
equilibrium price, Lemma 6 provides sign information on the derivatives of both $p$ and $n$ with respect to the main parameters of the model, $\theta_{0}, \theta_{1}$, and $a$. Finally, Theorem 2 shows that profit per firm falls as the number of firms in the market increases (the total derivative of $\pi$ with respect to $n$ is negative), except possibly on a small interval of values of $\theta_{0}, \theta_{1}$, and $a$. Throughout this section we treat $n$ as a continuous variable for analytical convenience. Furthermore, when $n$ is permitted to change, $a$ is assumed to remain constant which means that as the number of firms is (say) increased, the size of the market is increased commensurately.

Lemma 4 The derivative of the symmetric equilibrium price is monotone in $n$; specifically, $d p / d n$ is positive when $\theta_{1} a>\theta_{0}>\theta_{1} a / 3$ and otherwise it is negative. The equation $p a-F=0$ implicitly defines the function $n=N\left(a, t, \theta_{0}, \theta_{1}, F\right)$.

Proof. Differentiating equation (27) with respect to $n$ we obtain

$$
\frac{d p}{d n}=\left(\theta_{0}-\frac{\theta_{1} a}{3}\right) \frac{d \omega_{1}^{\prime}}{d n}
$$

Recall $\omega_{1}^{\prime}$ is increasing in absolute value in $n$ so that $d \omega_{1}^{\prime} / d n$ has the same sign as $\omega_{1}^{\prime}$ and $\mu$. For $\mu>0, d \omega_{1}^{\prime} / d n>0$ and the sign of $d p / d n$ is the sign of the term in parentheses. For $\mu<0$ the term in parentheses is positive, making $d p / d n<0$.

The effect of a change in $t, \theta_{0}$, or $\theta_{1}$ on $n=N\left(a, t, \theta_{0}, \theta_{1}\right)$ is,

$$
\frac{d N}{d \theta_{0}}=-\frac{\partial p / \partial \theta_{0}}{\partial p / \partial n}
$$

and

$$
\frac{d N}{d a}=-\frac{p+a \partial P / \partial a}{a \partial p / \partial n}
$$


Lemma 5 For all $\mu \in(-0.5,0.5)$,

$$
\frac{d \omega_{1}^{\prime}}{d \mu}>0
$$

Lemma 6 If $\theta_{1} a / 3<\theta_{0}<\theta_{1} a$ (which implies $\mu>0$ )

\begin{tabular}{l|l|l|l|}
$\alpha$ & $\theta_{0}$ & $\theta_{1}$ & $a$ \\
\hline$\frac{d p}{d \alpha}$ & - & + & + \\
\hline$\frac{d N}{d \alpha}$ & + & - & - \\
\hline
\end{tabular}

If $\theta_{0}>\theta_{1} a$ (which is equivalent to $\mu<0$ )

\begin{tabular}{l|l|l|l|l|}
$\alpha$ & $\theta_{0}$ & $\theta_{1}$ & $a$ & $t$ \\
\hline$\frac{d p}{d \alpha}$ & - & + & + & + \\
\hline$\frac{d N}{d \alpha}$ & - & + & + & + \\
\hline
\end{tabular}

Proof. The proof is omitted; it is easy to verify each item.

For a circle of fixed circumference $\ell$ it is shown below in Lemma 2 that equilibrium profit per firm declines as $n$ increases.

Theorem 2 For $n \geq 2$ and a circle of fixed circumference $\ell$ (making $a=\ell /(2 n+1)$ ) equilibrium profit falls as $n$ rises for $\theta_{0} \geq \theta_{1} a$ and for $\theta_{0} \leq \theta_{1} a / 3$.

Proof. The derivative of profit with respect to the number of firms is

$$
\frac{d \pi}{d n}=a \frac{\partial p}{\partial n}+\left[p+a \frac{\partial p}{\partial a}+a \frac{\partial p}{\partial \omega_{1}^{\prime}} \frac{\partial \omega_{1}^{\prime}}{\partial \mu} \frac{d \mu}{d a}\right] \frac{d a}{d n}
$$

It is easily determined that (i) $d a / d n<0$, (ii) $\partial p / \partial \omega_{1}^{\prime}=a\left(\theta_{0}-\theta_{1} a / 3\right) / 2$, (iii) $\partial \omega_{1}^{\prime} / \partial \mu>0$, (iv) $\partial p / \partial a>0,(\mathrm{v})$ and $\partial \mu / \partial a>0$.

Take first the case of $\theta_{0} \geq \theta_{1} a$. We know that $d a / d n<0$ and from Lemma 4 $\partial p / \partial n<0$; therefore, $d \pi / d n<0$ if the square bracket term in equation (32) is non- 
negative. Clearly both $p$ and $\partial p / \partial a$ are positive, and, as $\theta_{0} \geq \theta_{1} a$, then $\partial p / \partial \omega_{1}^{\prime} \geq 0$ so that all square bracket terms are non-negative and $d \pi / d n<0$.

Now consider $\theta_{0}<\theta_{1} a$; then $\mu>0$. Using $r_{2} r_{1}=1$,

$$
r_{2}-r_{1}=2 \sqrt{\frac{1}{4 \mu^{2}}-1}
$$

and

$$
2 a t=\frac{\left(\theta_{1} a-\theta_{0}\right)(1-2 \mu)}{\mu}
$$

$d \pi / d n$ is

$$
\begin{gathered}
\frac{d \pi}{d n}=\frac{-a^{2}}{2 n+1}\left(6 a t+9 \theta_{1} a-6 \theta_{0}+2 \theta_{0} \omega_{1}^{\prime}-\theta_{1} a \omega_{1}^{\prime}\right) \\
+a^{2}\left(\theta_{0}-\frac{\theta_{1} a}{3}\right)\left[\frac{\left(r_{2}-r_{1}\right)\left(\ln r_{2}-\ln r_{1}\right)}{2\left(r_{2}^{n}-r_{1}^{n}\right)^{2}}-\frac{2 t a \theta_{0}}{(2 n+1)\left(\theta_{1} a-\theta_{0}\right)^{2}} \sum_{j=0}^{n-1}\left(\frac{r_{2}^{j}-r_{1}^{j}}{r_{2}^{n}-r_{1}^{n}}\right)^{2}\right]
\end{gathered}
$$

Using $6 a t+9 \theta_{1} a-6 \theta_{0}+2 \theta_{0} \omega_{1}^{\prime}-\theta_{1} a \omega_{1}^{\prime}<6\left(t+\theta_{1}\right) a$ and $\left(r_{2}-r_{1}\right)\left(\ln r_{2}-\ln r_{1}\right) /\left(r_{2}^{n}-r_{1}^{n}\right)^{2}>$ $0, d \pi / d n<0$ is implied by

$$
6\left(t+\theta_{1}\right) a \geq \frac{2 t \theta_{0} a\left(\theta_{0}-\frac{\theta_{1} a}{3}\right)}{\left(\theta_{1} a-\theta_{0}\right)^{2}} \sum_{j=0}^{n-1}\left(\frac{r_{2}^{j}-r_{1}^{j}}{r_{2}^{n}-r_{1}^{n}}\right)^{2}
$$

Using $r_{1}=r_{2}^{-1}$

$$
\begin{gathered}
\sum_{j=0}^{n-1}\left(\frac{r_{2}^{j}-r_{1}^{j}}{r_{2}^{n}-r_{1}^{n}}\right)^{2}=\frac{r_{2}^{2 n}-r_{2}^{-2(n-1)}+r_{2}^{2}-1}{\left(r_{2}^{n}-r_{2}^{-n}\right)^{2}\left(r_{2}^{2}-1\right)}-\frac{2 n}{\left(r_{2}^{n}-r_{2}^{-n}\right)^{2}} \\
=\frac{r_{2}^{2 n}-2+r_{2}^{-2 n}}{\left(r_{2}^{n}-r_{2}^{-n}\right)^{2}\left(r_{2}^{2}-1\right)}-\frac{r_{2}^{2 n}-2+r_{2}^{2(n-1)}}{\left(r_{2}^{n}-r_{2}^{-n}\right)^{2}\left(r_{2}^{2}-1\right)}-\frac{2 n}{\left(r_{2}^{n}-r_{2}^{-n}\right)^{2}} \\
>\frac{1}{r_{2}^{2}-1}
\end{gathered}
$$


Then equation (33) and thus $d \pi / d n<0$ is implied by

$$
6\left(t+\theta_{1}\right) \geq \frac{2 t \theta_{0}\left(\theta_{0}-\frac{\theta_{1} a}{3}\right)}{\left(r_{2}^{2}-1\right)\left(\theta_{1} a-\theta_{0}\right)^{2}}
$$

Using

$$
\begin{aligned}
r_{2}^{2}-1 & =\frac{1}{2 \mu^{2}}+\sqrt{0.25-\mu^{2}}-2 \\
& >\frac{1}{2 \mu^{2}}-2=\frac{2 a^{2} t^{2}+4 a t\left(\theta_{1} a-\theta_{0}\right)}{\left(\theta_{1} a-\theta_{0}\right)^{2}}
\end{aligned}
$$

equation (33) is implied by

$$
6\left(t+\theta_{1}\right) a \geq \frac{\theta_{0}\left(\theta_{0}-\frac{\theta_{1} a}{3}\right)}{a^{2} t+2 a\left(\theta_{1} a-\theta_{0}\right)}
$$

Maximizing the numerator of the right hand side of equation (34) by setting $\theta_{0}=$ $\theta_{1} a / 6$ and minimizing the denominator by setting $\theta_{0}=0$, the right hand side is bounded above by

$$
\frac{\theta_{1}^{2}}{6 t+12 \theta_{1}}
$$

and hence $d \pi / d n<0$ is implied by

$$
1 \geq \frac{\theta_{1}^{2}}{36\left(t+\theta_{1}\right)\left(t+2 \theta_{1}\right)}
$$

which clearly holds.

For the case of $\theta_{0} \theta_{1} a / 3 \leq \theta_{0} \leq \theta_{1} a$ it remains possible that $d \pi / d n>0$. We have verified by a examples that $d \pi / d n>0$ can occur. ${ }^{4}$

\footnotetext{
${ }^{4}$ Let $\theta_{0}=\lambda \theta_{1} a$ for some value of $\lambda \in(0,1 / 3)$. Then $\mu$ does not depend on $a$ nor do $r_{1}$ and $r_{2}$. The expression for $d \pi / d n$ is comprised of three clusters of terms; the first two are negative and the third is positive. The positive term is multiplied by $a$ to a lower power than the other two; therefore, one may choose $a$ very small and obtain the desired result.
} 


\section{A Related Model and Welfare}

In this section we find the welfare optimal number of firms in the market, ignoring the fixed cost $F$. We also examine another model in which the consumption externality depends on the number of consumers buying the same good, but which does not depend on the consumers' identities. The two models have identical equilibrium conditions when firms' markets are connected segments; however, the welfare optimal number of firms is not the same. The alternate model is sketched next. Following that, the welfare optima are found.

Imagine a model in which consumers are affected by the number of others who consume the same good, but do not care who those other consumers are. This can be modeled with utility given by

$$
u_{y}^{i}\left(p_{i}, \underline{z}_{i}, \bar{z}_{i}\right)=K-p_{i}-t\left(y-\ell_{i}\right)^{2}+\theta_{0}\left(\bar{z}_{i}-\underline{z}_{i}\right)-\frac{\theta_{1}}{2}\left(\bar{z}_{i}-\underline{z}_{i}\right)^{2}
$$

when the consumer is at $y$, the firm is at $\ell_{i}$, and the market served by the firm is at $\left[\underline{z}_{i}, \bar{z}_{i}\right)$. As with the model analyzed throughout this paper, the consumer gains as more people consume the same good, but the gain is falling on the margin. Eventually a level of saturation is reached and further expansion of the market reduces the consumer's utility. The difference here is that only numbers count; the externality does not depend on the location of the cohort consuming the good. Of course, the presence of the element measuring the distance between the consumer and the good she buys suggests that firms are likely to have connected markets.

In the model analyzed throughout the paper recall that the conditions defining market boundaries depend on the utility of the consumer at the market boundary. 
At a symmetric equilibrium this utility is

$$
K-p-\frac{t}{2} a^{2}+\theta_{0} a-\frac{\theta_{1}}{2} a^{2}
$$

which is precisely the value of equation (35) for the consumer on the boundary of two markets when all firms charge $p$ and have connected market areas of size $a$. The two models yield the same equilibrium.

Welfare, however, is another matter. In the model in which consumers care who also consumes the good, the equilibrium utility of the consumer located at $y$ is

$$
U^{*}(y, p)=K-p+\theta_{0} a-\left(\frac{t+2 \theta_{1}}{4}\right) a^{2}+\left(t+\theta_{1}\right) a y-\left(t+\theta_{1}\right) y^{2}
$$

and the total welfare of the firm plus all consumers served by the firm is

$$
W T=p a-F+\int_{0}^{a} U^{*}(y, p) d y=K a+\theta_{0} a^{2}-\left(\frac{t+4 \theta_{1}}{12}\right) a^{3}-F
$$

The average total welfare is

$$
W=\frac{W T}{a}=K+\theta_{0} a-\left(\frac{t+4 \theta_{1}}{12}\right) a^{2}-\frac{F}{a}
$$

If $F=0$, then $W$ is maximized at

$$
a=\frac{6 \theta_{0}}{t+4 \theta_{1}}
$$

For $F>0$, equation (36) has a maximum corresponding to the unique real root of the first order condition.

Performing a parallel welfare calculation for the alternate model yields

$$
a=\frac{6 \theta_{0}}{t+6 \theta_{1}}
$$


This value satisfies equation (14), the contraction condition, if $t+6 \theta_{1} \leq 2 t+3 \theta_{1}$ or $3 \theta_{1} \leq t$

From equation (36) it is easily seen that the externality makes a positive contribution to the average per capita welfare at the optimum if and only if $\theta_{0}>\theta_{1} a / 3$.

\section{Conclusion}

We have analyzed a generalized Salop [5] model that features a consumption externality under which each consumer's utility rises when similar consumers buy the same good and falls when sufficiently dissimilar consumers buy the good. Conditions for existence of a symmetric equilibrium are found and the equilibrium is shown to be unique. The welfare optimum number of firms is found and it is seen that, generally, but not universally, equilibrium profit per firm falls as the number of firms rises.

The utility structure of the model captures goods such as social clubs and housing neighborhoods where a person typically wishes to consume the same thing as people who are similar to her. That is, the good modeled here has a "birds of a feather flock together" character. We also look at a variant of the model under which the utility of a consumer depends on the number, but not the identity, of consumers buying the same good. This is similar to Pesendorfer [4]; however, the focus of his work and ours materially differ. He has two types of consumer, one firm, and is interested in the timing of new designs marketed by the firm. We, on the other hand, have a continuum of consumers and a fixed, arbitrary number of firms. We want to see how consumers sort themselves among firms. In our model with anonymity, the symmetric equilibrium is identical to that of the non-anonymous version, but the welfare optimal number of firms is greater. 


\section{References}

[1] Laurie Simon Bagwell and B. Douglas Bernheim. Veblen effects in a theory of conspicuous consumption. American Economic Review, 86:349-373, 1996.

[2] Claude d'Aspremont, Jean J. Gabszewicz, and Jacques-François Thisse. On hotelling's 'stability in competition'. Econometrica, 47:1145-1150, 1979.

[3] Michael L. Katz and Carl Shapiro. Systems competition and network effects. Journal of Economic Perspectives, 8:93-115, 1994.

[4] Wolfgang Pesendorfer. Design innovation and fashion cycles. American Economic Review, 85:771-792, 1995.

[5] Steven C. Salop. Monopolistic competition with outside goods. Bell Journal of Economics, 10:141-156, 1979.

[6] Thorstein Veblen. The Theory of the Leisure Class. Kelley, New York, 1965 [1899]. 


\section{Appendix}

\section{A Equilibrium When $\theta_{1}=0$}

Investigation of the function $\phi$ when $\theta_{1}=0$ shows that the functions $\omega_{i}$ are linear and can be written $\omega_{i}\left(x_{0}\right)=\alpha_{i}+\rho_{i} x_{0}$ where

$$
\rho_{i} \equiv \frac{r_{2}^{n-i}-r_{1}^{n-i}}{r_{2}^{n}-r_{1}^{n}}
$$

If $\theta_{1}=0$ then

$$
\mu=\frac{-\theta_{0}}{2 a t-2 \theta_{0}}
$$

The condition $\mu \in(-0.5,0.5)$ requires $a>2 \theta_{0} / t$ which, in turn implies that $\mu<0$. The value of $\alpha_{i}$ is readily found by recalling that $\omega_{i}(a / 2)=\alpha_{i}+\rho_{i} a / 2=a / 2$; therefore,

$$
\omega_{i}\left(x_{0}\right)=\frac{a}{2}\left(1-\rho_{i}\right)+\rho_{i} x_{0}
$$

Equation (15) becomes

$$
P\left(p, x_{0}\right)=p-\theta_{0}\left[\frac{a}{2}\left(1-\rho_{1}\right)+\rho_{1} x_{0}\right]+a^{2} t-\theta_{0} a-\left(2 a t-3 \theta_{0}\right) x_{0}
$$

and the equilibrium price is

$$
p=\frac{a}{2}\left[2 a t-\left(3-\rho_{1}\right) \theta_{0}\right]
$$

The second order condition for profit maximization is

$$
\frac{d^{2} \pi_{0}}{d x_{0}^{2}}=4\left(\frac{\partial P}{\partial x_{0}}+\frac{\partial P}{\partial x_{1}} \omega_{1}^{\prime}\right)=-4\left[2 a t-\left(3-\rho_{1}\right) \theta_{0}\right]
$$


Noting that $\rho_{1} \in(-1,0)$, the second order condition is negative if $a>2 \theta_{0} / t$. This is the same condition that guarantees $|\mu|<0.5$ making $\phi$.a contraction and causing $\rho_{1} \in(-1,0)$. Superficially $a>2 \theta_{0} / t$ appears in conflict with the condition

$$
a>\frac{6 \theta_{0}}{2 t+3 \theta_{1}}
$$

that is used in Lemma 2 to ensure the contraction property. The seeming inconsistency is because the latter condition is only sufficient and is loose in the sense that it makes no attempt to use any of the structure of market allocations beyond $x_{i} \in[0, a]$.

\section{B Proof of Lemma}

\section{Proof.}

$$
\begin{aligned}
\left(r_{2}^{n}-r_{1}^{n}\right)^{2} \frac{d \omega_{1}^{\prime}}{d \mu}= & (n-1)\left(r_{2}^{n-2} \frac{d r_{2}}{d \mu}-r_{1}^{n-2} \frac{d r_{1}}{d \mu}\right)\left(r_{2}^{n}-r_{1}^{n}\right) \\
& -n\left(r_{2}^{n-1} \frac{d r_{2}}{d \mu}-r_{1}^{n-1} \frac{d r_{1}}{d \mu}\right)\left(r_{2}^{n-1}-r_{1}^{n-1}\right)
\end{aligned}
$$

Recalling that $r_{1} r_{2}=1$ and hence

$$
\frac{d r_{2} / d \mu}{d r_{1} / d \mu}=-r_{2}^{2}
$$


so that, setting $m=2 n-1$,

$$
\begin{aligned}
\frac{\left(r_{2}^{n}-r_{1}^{n}\right)^{2}}{r_{2} d r_{1} / d \mu} \frac{d \omega_{1}^{\prime}}{d \mu} & =r_{2}^{m}-r_{2}^{-m}-m\left(r_{2}-r_{2}^{-1}\right)=\frac{r_{2}^{2 m}-1}{r_{2}^{m}}-m \frac{r_{2}^{2}-1}{r_{2}} \\
& =\frac{r_{2}^{2}-1}{r_{2}^{m}}\left(r_{2}^{2 m-2}+r_{2}^{2 m-4}+\ldots+1-m r_{2}^{m-1}\right) \\
& =\frac{r_{2}^{2}-1}{r_{2}^{m}} \sum_{j=0}^{m-1}\left(\frac{r_{2}^{2(m-1-j)}+r_{2}^{2 j}}{2}-r_{2}^{m-1}\right) \\
& =\frac{r_{2}^{2}-1}{2 r_{2}} \sum_{j=0}^{m-1}\left(r_{2}^{m-1-2 j}+r_{2}^{-(m-1-2 j)}-2\right)
\end{aligned}
$$

The expression in parentheses in the last line of equation (37) is easily shown to be positive by noting that it has the form $x+x^{-1}-2$ where $x=r_{2}^{2(n-j-1)}>0$. The latter is uniquely minimized at $x=1$ where its value is zero. Thus

$$
\frac{d \omega_{1}^{\prime}}{d \mu}=\frac{r_{2}^{2}-1}{2\left(r_{2}^{n}-r_{1}^{n}\right)^{2}} \frac{d r_{1}}{d \mu} \sum_{j=0}^{m-1}\left(r_{2}^{m-1-j}+r_{2}^{-(m-1-j)}-2\right)
$$

and the sign of $d \omega_{1}^{\prime} / d \mu$ is positive, because the sign of both $r_{2}^{2}-1$ and $d r_{1} / d \mu$ are the same as the sign of $\mu$ making their product positive. 